\title{
Influence of shell effects on mass asymmetry in fission of different $\mathrm{Hg}$ isotopes
}

\author{
A.V. Andreev, G.G. Adamian, and N.V. Antonenko \\ Bogoliubov Laboratory of Theoretical Physics, Joint Institute for Nuclear Research, 141980 Dubna, \\ Russia
}

\begin{abstract}
With the improved scission-point model mass distributions are calculated for induced fission of $\mathrm{Hg}$ isotopes with even mass numbers $\mathrm{A}=174-198$. The calculated mass distributions and mean total kinetic energy of fission fragments are in good agreement with the existing experimental data. The asymmetric mass distribution of fission fragments of ${ }^{180} \mathrm{Hg}$ observed in the recent experiment is explained. The change in the shape of the mass distribution with increasing $\mathrm{A}$ of the fissioning ${ }^{A} \mathrm{Hg}$ nucleus from symmetric for ${ }^{174} \mathrm{Hg}$ to asymmetric around ${ }^{180} \mathrm{Hg}$, and to more symmetric for ${ }^{192-198} \mathrm{Hg}$ is revealed.
\end{abstract}

The interest to the theoretical exploration of fission process was re-stimulated several years ago after the experiment on $\beta$-delayed fission of ${ }^{180} \mathrm{Tl}[1,2]$, where the mass distribution of fission fragments in the fission of the post- $\beta$-decay daughter nucleus ${ }^{180} \mathrm{Hg}$ was found to be strongly asymmetric. That was surprising since from the previous investigations the mass distributions were expected to be symmetric for the nuclei lighter than thorium. Some indications of slight asymmetry were observed earlier in the experiments on fission of Au-Po nuclei [3], but the difference of the mass distribution shape from symmetric Gaussian shape was rather small. The experiment [1] was followed by several theoretical works describing the observed asymmetric shape of the fission-fragment mass distribution [4-7]. The present work is the extension of our previous calculations [4] to the wider range of $\mathrm{Hg}$ isotopes in order to explore the fission fragment mass distribution shape dependence on the mass number $A$ of the fissioning ${ }^{A} \mathrm{Hg}$ isotope and compare with the results of other theoretical models.

We describe the nuclear fission observables within the improved scission-point model $[4,8]$ which operates with relative probabilities of formation of different scission configurations obtained with statistical approach by calculating the potential energy of scission configurations. The fissioning nucleus at the scission point is modeled by a dinuclear system consisting of two nearly touching prolate coaxial spheroids with deformation parameters defined as the ratios of the major and minor semi-axes of the spheroids $\beta_{i}=c_{i} / a_{i}$, where $i=L, H$ denotes light and heavy fragments of the dinuclear system, respectively. The mass and charge numbers of the fragments are $A_{i}$ and $Z_{i}$, respectively. $A=A_{L}+A_{H}$ and $Z=Z_{L}+Z_{H}$ are the mass and charge numbers of the fissioning nucleus, respectively. The potential energy (1) of the dinuclear system consists of the energies of the fragments, energy of their interaction, and rotational energy $V^{r o t}$ in case of induced fission. The energy of each fragment consist of the liquid-drop energy $U_{i}^{L D}$, deformation dependent shell correction term $\delta U_{i}^{\text {shell }}$, and the energy of

This is an Open Access article distributed under the terms of the Creative Commons Attribution License 2.0, which permits unrestricted use, distribution, and reproduction in any medium, provided the original work is properly cited. 
the zero-point vibrations $U_{i}^{z p v}$, associated with the energy $E^{2^{+}}$of the first $2^{+}$excited state. The damping of the shell corrections with temperature is introduced in order to take into account the influence of the excitation energy. The interaction potential consists of the Coulomb interaction potential $V^{C}$ of the two uniformly charged spheroids and nuclear interaction potential $V^{N}$ in the form of a double folding of Woods-Saxon nuclear densities of the fragments and Skyrme-type density-dependent nucleon-nucleon interaction. The interaction potential has a pocket at a distance between the tips of the fragments of about 0.5-1 fm (in the considered region of fissioning nuclei) depending on deformations of the fragments. The internuclear distance $R$ is taken corresponding to the position of this pocket (minimum) $R=R_{m}\left(\beta_{i}\right.$ ). The excitation energy $E^{*}$ is calculated as the initial excitation energy of the fissioning nucleus plus the difference between the potential energy of the fissioning nucleus and the dinuclear system at the scission point. The details of the calculations of the potential energy can be found in [4].

$$
\begin{aligned}
U\left(A_{i}, Z_{i}, \beta_{i}, R, l\right)= & U_{L}^{L D}\left(A_{L}, Z_{L}, \beta_{L}\right)+\delta U_{L}^{\text {shell }}\left(A_{L}, Z_{L}, \beta_{L}\right)+U_{L}^{z p v}\left(A_{L}, Z_{L}\right) \\
& +U_{H}^{L D}\left(A_{H}, Z_{H}, \beta_{H}\right)+\delta U_{H}^{\text {shell }}\left(A_{H}, Z_{H}, \beta_{H}\right)+U_{H}^{z p v}\left(A_{H}, Z_{H}\right) \\
& +V^{C}\left(A_{i}, Z_{i}, \beta_{i}, R\right)+V^{N}\left(A_{i}, Z_{i}, \beta_{i}, R\right)+V^{r o t}\left(A_{i}, Z_{i}, \beta_{i}, R, l\right), \\
R= & R_{m}\left(A_{i}, Z_{i}, \beta_{i}, l\right) .
\end{aligned}
$$

The relative probability of formation of a dinuclear system with particular masses, charges and deformations of the fragments is calculated within the statistical approach as follows:

$$
Y\left(A_{i}, Z_{i}, \beta_{i}, l\right) \sim \exp \left\{-\frac{U\left(A_{i}, Z_{i}, \beta_{i}, l\right)}{T(l)}\right\} .
$$

The temperature is $T(l)=\sqrt{E^{*}(l) / a}$, where $a=A / 12$ is the level density parameter. In order to obtain the mass distribution in fission of a particular nucleus with the mass number $A$ and charge number $Z$ one should integrate (2) over $Z_{L}, \beta_{L}$, and $\beta_{H}$, sum over $l$, and take into account that $A_{L}+A_{H}=A$ and $Z_{L}+Z_{H}=Z$. Finally, for the calculation of mass distribution the following expression is obtained:

$$
Y\left(A_{L}\right)=\frac{\sum_{l=0}^{l_{\max }}(2 l+1) \int \exp \left\{-\frac{U\left(A_{i}, Z_{i}, \beta_{i}, l\right)}{T(l)}\right\} \mathrm{d} Z_{L} \mathrm{~d} \beta_{L} \mathrm{~d} \beta_{H}}{\sum_{l=0}^{l_{\max }}(2 l+1) \int \exp \left\{-\frac{U\left(A_{i}, Z_{i}, \beta_{i}, l\right)}{T(l)}\right\} \mathrm{d} A_{L} \mathrm{~d} Z_{L} \mathrm{~d} \beta_{L} \mathrm{~d} \beta_{H}} .
$$

This distribution is normalized to unity.

For comparison with the existing experimental data we made calculations for the fission of post - $\beta$-decay daughter nucleus ${ }^{180} \mathrm{Hg}$ having the excitation energy of $10.44 \mathrm{MeV}$ (Fig. 1) and for the fission of ${ }^{198} \mathrm{Hg}$ obtained in reactions: ${ }^{197} \mathrm{Au}(p, \mathrm{f})$ at $E_{p}=22.4 \mathrm{MeV}$ and ${ }^{194} \mathrm{Pt}(\alpha, \mathrm{f})$ at $E_{\alpha}=50.4 \mathrm{MeV}$ (Fig. 2). The results for ${ }^{180} \mathrm{Hg}$ are compared with the experimental data [2]. In agreement with the experiment we obtained asymmetric mass distribution. The calculated average mass of the light fragment is $\overline{A_{L}}=179$ with $\sigma_{A_{L}}=5$. The kinetic energy was calculated by assuming that all the interaction energy at the scission point transforms into the kinetic energy after large separation of the fission fragments. The obtained value of $136 \mathrm{MeV}$ is in good agreement with the experimental observation. For ${ }^{198} \mathrm{Hg}$ we obtained more symmetric mass distribution with a dip at the top, which becomes smaller with excitation, that is in agreement with the experimental data [9].

For the illustration of the dependence of the mass distribution shape on the mass of the fissioning $\mathrm{Hg}$ isotope we calculated the mass distributions for fission of isotopes ${ }^{174-196} \mathrm{Hg}$ with even mass numbers at zero angular momentum $l=0$. To show the dependence on excitation the calculations were made 


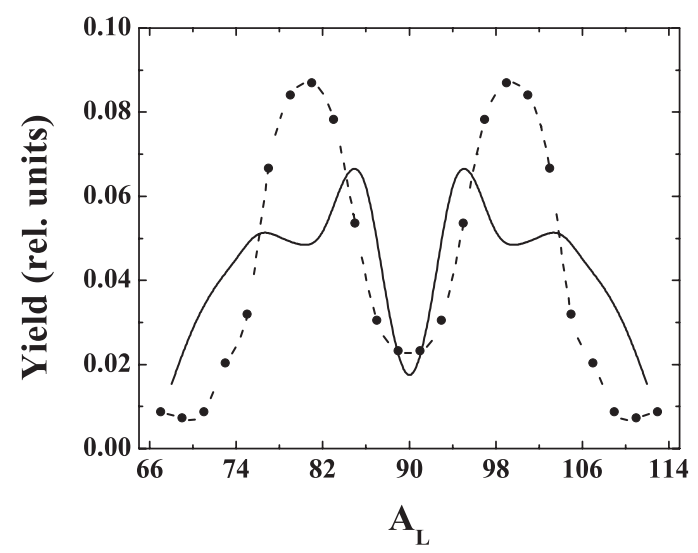

Figure 1. Calculated (solid line) and experimental [2] (points connected by dashed line) mass distributions of fission fragments for $\beta$-delayed fission of ${ }^{180} \mathrm{Tl}$ (fissioning nucleus is ${ }^{180} \mathrm{Hg}$ ).

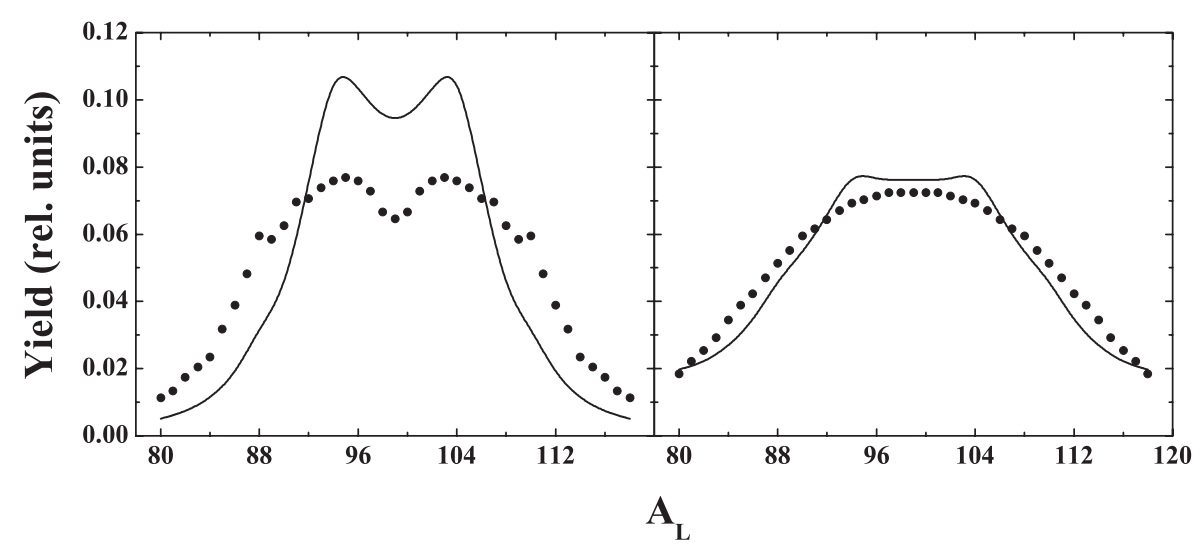

Figure 2. Calculated (solid line) mass distribution of fission fragments in comparison with the experimental data [9] (points) for induced fission of ${ }^{198} \mathrm{Hg}$ in the reactions ${ }^{197} \mathrm{Au}(\mathrm{p}, \mathrm{f})$ at $E_{p}=22.4 \mathrm{MeV}$ (left panel) and ${ }^{194} \mathrm{Pt}(\alpha, \mathrm{f})$ at $E_{\alpha}=50.4 \mathrm{MeV}$ (right panel).

at two excitation energies $2 \mathrm{MeV}$ and $20 \mathrm{MeV}$ above the fission barriers. The results are presented in Figures 3 and 4 . For ${ }^{174} \mathrm{Hg}$ the distribution has a symmetric shape. With increasing mass number of $\mathrm{Hg}$ the asymmetry appears and becomes maximal for ${ }^{180-182} \mathrm{Hg}$. Further from ${ }^{182} \mathrm{Hg}$ to ${ }^{190} \mathrm{Hg}$ the dip at symmetric mass division remains, but the asymmetry becomes smaller due to decrease of the width of the distribution. From ${ }^{190} \mathrm{Hg}$ to ${ }^{196} \mathrm{Hg}$ the distribution remains narrow while the dip at symmetric mass division diminishes. With increasing excitation the asymmetry decreases.

In order to explain such a behavior of the mass distribution shape one can analyse a deformation dependence of the potential energy on the deformations of the fragments at the scission point. It is well known that the shell effects are responsible for the appearance of the asymmetric mass distributions. Due to strong Coulomb repulsion the potential energy (1) will always have a minimum at deformations of about $\beta_{i}=1.5-1.8$, that is larger than the ground state deformations of the nuclei-fragments. Hence, the shell effects at large deformations mostly influence the mass distribution shape while the shell effects around the ground state deformations of the fragments does not play important role. 


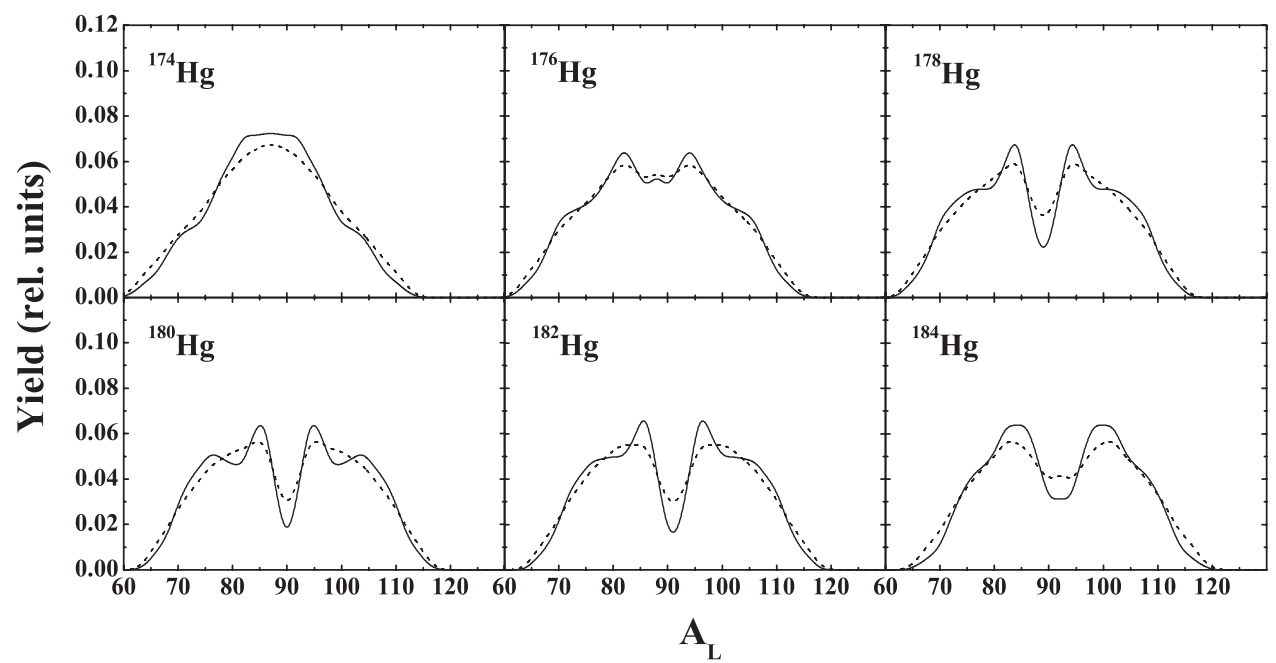

Figure 3. Calculated mass distributions for the fission of ${ }^{174-184} \mathrm{Hg}$ at $l=0$ with the excitation energies $2 \mathrm{MeV}$ (solid line) and $20 \mathrm{MeV}$ (dashed line) above the fission barrier.

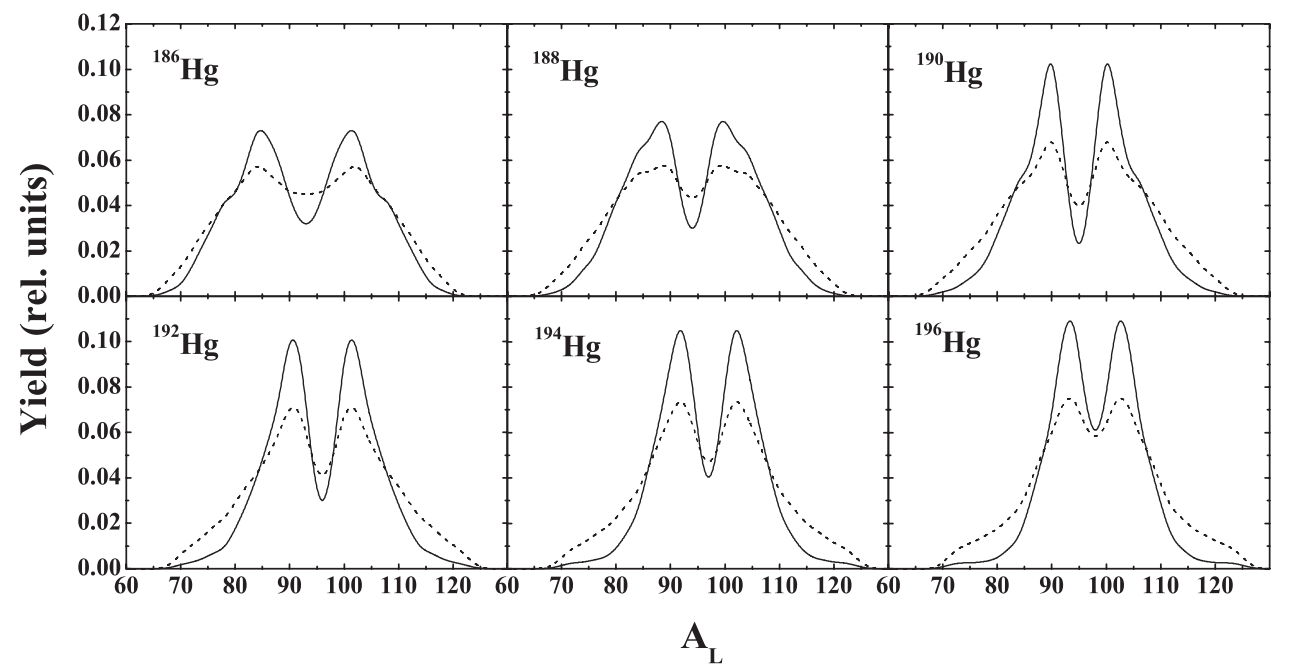

Figure 4. The same as in Figure 3, but for the fission of ${ }^{186-196} \mathrm{Hg}$.

In the vicinity of the symmetric mass division of ${ }^{180} \mathrm{Hg}$, the shell effects in the fragments close to the magic nucleus ${ }^{90} \mathrm{Zr}$ are strongly negative at small deformations, but at the deformations around the liquid-drop minimum of the potential energy at the region of large deformations the shell corrections are positive. That is why the presence of magic fragments does not lead to a large yield of symmetric mass split in fission of ${ }^{180} \mathrm{Hg}$. However, the rigidity of a magic ${ }^{90} \mathrm{Zr}$ lead to a very narrow minimum on the potential energy surface as a function of deformations for the scission configurations ${ }^{90} \mathrm{Zr}+{ }^{90} \mathrm{Zr}$ and around (see [4]). Due to integration in (3) such a narrow minimum leads to a smaller yield of this mass split. The other factor leading to decrease of the yield of a symmetric mass split in fission of ${ }^{180} \mathrm{Hg}$ and nearby isotopes is the high $E^{2+}$ values around ${ }^{90} \mathrm{Zr}$ contributing to the term $E^{z p v}$ in (1). 
For the asymmetric mass split of ${ }^{180} \mathrm{Hg}$, the shell effects are rather weak and the potential energy as a function of fragment deformations has a wide minimum, that will lead to larger yield of the asymmetric split. Also, the values of $E^{2+}$ for the fragments are small. All the above described factors results in the asymmetric shape of the mass distribution in fission of ${ }^{180} \mathrm{Hg}$ and nearby isotopes.

If we consider the isotopes of mercury lighter than ${ }^{180} \mathrm{Hg}$ the shell effects are rather weak for all mass divisions and we obtain a symmetric shape of the mass distribution. For the region of heavy mercury isotopes the influence of shell effects at symmetric mass split $\mathrm{Zr}+\mathrm{Zr}$ is not so strong as in ${ }^{90} \mathrm{Zr}+{ }^{90} \mathrm{Zr}$, however, the influence of a semi-magic proton number $Z=40$ for zirconium results in a dip at symmetric mass split similar to that in ${ }^{180} \mathrm{Hg}$. The depth of this dip decreases with increasing mass of the fissioning mercury isotope.

Our model gives a good description of the existing experimental data on fission of ${ }^{180} \mathrm{Hg}$ and ${ }^{198} \mathrm{Hg}$. We predicted the change of the shape of the mass distribution with increasing mass number of the fissioning mercury isotope from symmetric for ${ }^{174} \mathrm{Hg}$ to asymmetric around ${ }^{180} \mathrm{Hg}$ and to more symmetric for ${ }^{192-196} \mathrm{Hg}$. The key role in description of the mass asymmetry in our model play the deformation dependent shell effects in the fission fragments.

We thank Dr. T. M. Shneidman for fruitful discussions. This work was supported by RFBR (Moscow) and JINR grant No. 13-302-01.

\section{References}

[1] A. N. Andreyev et al., Phys. Rev. Lett. 105, 252502 (2010)

[2] J. Elseviers et al., submitted to Phys. Rev. C

[3] M. G. Itkis, N. A. Kondrat'ev, S. I. Mul'gin, V. N. Okolovich, A. Ya. Rusanov, and G. N. Smirenkin, Sov. J. Nucl. Phys. 53, 757 (1991)

[4] A. V. Andreev, G. G. Adamian, and N. V. Antonenko, Phys. Rev. C 86, 044315 (2012)

[5] P. Möller, J. Randrup, and A. J. Sierk, Phys. Rev. C 85, 024306 (2012)

[6] M. Warda, A. Staszczak, and W. Nazarewicz, Phys. Rev. C 86, 024601 (2012)

[7] S. Panebianco, J.-L. Sida, Héloise Goutte, J.-F. Lemaître, Noël Dubray, and Stéphane Hilaire, Phys. Rev. C 86, 064601 (2012)

[8] A. V. Andreev, G. G. Adamian, N. V. Antonenko, S. P. Ivanova, and W. Scheid, Eur. Phys. J. A 22, 51 (2004)

[9] M. G. Itkis, N. A. Kondrat'ev, S. I. Mul'gin, V. N. Okolovich, A. Ya. Rusanov, and G. N. Smirenkin, Sov. J. Nucl. Phys. 52, 601 (1990) 